\title{
Documented Impact of Transportation Demand Management Programs Through the Case Study Method
}

\author{
Sara J. Hendricks and Nevine Labib Georggi \\ Center for Urban Transportation Research, University of South Florida
}

\begin{abstract}
What are the internal and external conditions of a work site that influence the success of its commuter choice program, aside from the trip-reducing services and incentives themselves? This article shares the results of two studies that used a more rigorous case study method for discerning the relative influence of conditions within and outside of the control of a work site. It also discusses the useful application and elements of the case study method as well as the location for finding properly documented case studies that describe successful programs. Recommendations are developed for work sites to implement commuter choice programs for TDM marketing professionals to identify receptive work-site candidates and for TDM researchers to investigate further using the case study method. By understanding the relative importance of various conditions, TDM professionals, employers, and employee transportation coordinators are better equipped to exert influence on factors within their control to develop more effective programs.
\end{abstract}




\section{Introduction}

A common transportation goal of urbanized areas is to reduce traffic congestion during a.m. and p.m. weekday peak hours. Programs that apply transportation demand (TDM) management strategies to individual employment work sites are one potentially effective response that has been used in urban areas throughout the United States over the last 25 years. Participating employers have offered programs and incentives to employees of work sites located in congested areas and who commute during times of peak traffic congestion. The programs are designed to persuade commuting employees to switch to a different travel mode other than single occupant vehicle (SOV), or to change travel time to outside the peak period, telecommute, decrease trip making, or decrease distance traveled.

Transportation professionals, program funding agencies, and employers want to know the level of effectiveness of these programs. TDM marketing specialists charged with promoting the creation of TDM programs need reliable information about how successful programs can be duplicated at other work sites and what factors contribute to TDM program success.

While numerous programs exist across the nation, the most abundant information includes traffic problem characterizations and simple descriptions of efforts undertaken. Many programs have not collected baseline information about prior commuting characteristics to compare against travel behavior after program implementation. Many programs have not measured program results or adequately tracked progress over time but instead provide only a "snapshot" writeup of program characteristics for one point in time. Often there is an inadequate demonstration of the causal link between the program and the results. Other programs may track data that are inadequate indicators of program effectiveness. There are many preconceived notions that arise from anecdotal information, not based upon any sound evidence, about what conditions are ideal for successful programs. For example, common guidance calls for establishing a work-site commuter choice program administered from the human resources department. This advice originated from observations about what many organizations have already done in the past rather than any evidence that human resources departments have special qualities for directing commuter choice programs. Another commonly held belief is that employee transportation coordinators (ETC) with charismatic personalities will be able to persuade commuters to alter their travel behavior. In addition to the limited usefulness of anecdotal information, the good quality program information that does exist is usually not shared but archived 
separately at each program source. The data are not gathered in one known place where they can be easily accessed and used by others.

The availability of properly documented TDM program results is critical to knowing what strategies work effectively and how to improve existing programs. Positive results provide justification for TDM program funding and can convince other employers to start programs of their own.

\section{Background}

Two events helped shape the focus of the research described in this article. First, the 2001 Association for Commuter Transportation (ACT) End-of-Year Survey indicated that the number one desire of TDM professionals was the provision of information on TDM-oriented statistics and case studies. The second event was the development of the Work Site Trip Reduction Model and Manual (WTRM; Winters and Perez 2004) by the Center for Urban Transportation Research (CUTR). The WTRM is a tool for TDM professionals to use for refining the effectiveness of commuter choice programs. Using a neural network built with thousands of "before" and "after" plans from employers, the model quantifies the change in average vehicle ridership (AVR) as a result of applying various combinations of commuter choice program elements. AVR is the number of people transported to a work site for every 100 vehicles. A surprising finding of the WTRM was that the commuter choice program incentives themselves directly explain only about 18 percent of the variance in effectiveness between one program and another. Our research question then became: "What is it that makes work-site trip reduction programs effective? What circumstances explain the other 82 percent?" By using employee data aggregated to the employer level, the WTRM method captures the many complex social and institutional influences that determine whether a commuter choice program is successful. However, this method does not separate out and make known what these influences are.

Both the need for a TDM case study database and unanswered questions about what other circumstances besides commuter choice program elements explain program effectiveness provided reasons to use a more rigorous case study method in two research studies, both funded by the National Center for Transit Research with sponsorship from the Florida Department of Transportation. Some large datasets containing multiyear performance data for work-site commuter choice programs also became available. The Washington State Department of Transpor- 
tation Commute Trip Reduction Program offered the use of its data for more than 1,300 work sites, starting with baseline data as early as 1993 and performance data as late as 2003. Both research studies discussed in this article used the Washington State dataset.

\section{The Case Study Method}

There are at least two meanings to the term "case study" as well as two very different outcome expectations. In the first meaning, as is commonly used in the TDM field, case studies are brief anecdotes of usually successful programs. TDM practitioners want to use case studies to be able to say that if organization A takes a particular action that yields a certain effect, then organization $B$ can take the same action and expect the same effect. Typically what happens is that organization B will (rightly) claim that the experience of organization $A$ does not apply because of various differences between organizations $A$ and $B$. The utility of case studies is limited by this, with ever-growing requests for more case studies that better mirror the conditions of organization $\mathrm{B}$. The mirrored conditions are never close enough in similarity to create confidence that some action will yield the intended result.

The second meaning of case study is adapted from the social sciences. The foundation for a sound case study approach was articulated by Robert K. Yin of COSMOS Corporation in the early 1980s. This approach was adopted by the United States General Accounting Office (GAO) (United States General Accounting Office 1990), Program Evaluation and Methodology Division. ${ }^{1}$ Properly conceived case studies are a research method for attempting valid inferences from events outside the laboratory. In a lab, researchers can control for other variables to pinpoint the effect of a particular condition on the outcome. However, many study topics cannot be duplicated in a lab. These include complex human situations like work-site trip-reduction programs. In response, the case study method is applied for the purpose of examining the relationship between circumstances and outcome.

The case study method is one of several forms of inquiry used to collect and analyze empirical evidence (accumulated through observation). These also include surveys, experiments, archival analyses, and histories. All these methods can be used for exploratory, descriptive, and explanatory purposes. As outlined by Yin, case studies are used, as opposed to other research methods, when three general conditions are met: we want to learn how or why something is happening, the 
investigator has little control over events, and the focus is on contemporary events within circumstances that affect the outcome of those events (context). These conditions generally describe the circumstances under which researchers attempt to study work-site trip reduction programs.

Yin offers a definition of a case study as a research strategy. "A case study is an empirical inquiry that investigates a contemporary phenomenon within its real life context; when the boundaries between phenomenon and context are not clearly evident; and in which multiple sources of evidence are used" (Yin 1981). The GAO uses a close variation of this definition. The purpose behind multiple sources of evidence is that a hypothesis supported by one source of evidence is consistently reinforced (or disputed) by other independent information sources.

This second, more rigorous meaning of case study also has its own separate expectation as to outcome. Yin points out that one cannot use even the best executed case study to generalize to another population, such as organizations $A$ and $B$ (we also cannot generalize to another population based on one scientific experiment). However, we can generalize to a theoretical proposition or "rule of thumb." This is useful because attempting to apply the proposition will require one to acknowledge the influence of a unique context on the particular outcome.

There are several types of case studies, two of which are of interest to the topic of this article. An explanatory case study approach demonstrates a causal relationship between some variable and an outcome. An exploratory case study approach is more commonly used in the early stages of a subject under study. Often there are multiple variables of likely influence and not enough is known to define a more specific hypothesis confidently. Important to the case study process is the identification of explanations that rival a specified hypothesis. Thus, it is important to acknowledge and consider any other possible causes of the outcome under study, regardless of whether it supports the hypothesis. Efforts are made to search for evidence that supports the rival explanations as well as evidence that eliminates them from consideration.

In the development of a case study, there are clearly defined steps including the development of a research question, the articulation of a hypothesis, the selection of the type of case study best suited to the purpose of the researcher, development of the research design, data collection planning and implementation, analysis, and the reporting of findings. These steps were used in the two studies examining commuter choice programs in anticipation that in the long term the findings will be more robust, reliable, and useful in application. 


\section{The Relative Importance of Work-Site Culture}

The first of the two research studies using the case study method, "Commuter Choice Program Case Study Development and Analysis" (Hendricks and Joshi 2004), looked at work-site "organizational culture" as characterized by theorist Joanne Martin (Martin 2002) to determine if internal culture has some impact on trip reduction program success. Internal culture is an expansive topic and the research needed a more manageable focus within the practical constraints of the study. In response, researchers chose to study the impact of the designated "internal champion" or ETC as the assumed key representative of internal work-site culture within the context of the trip reduction program. A literature review had found that little research had been done on the topic of internal culture and ETCs in the past 10 years since Chen et al. (1995), Lopez-Aqueres (1993), and Wachs and Giuliano (1992).

Because little is known about the effect of organizational culture on travel behavior, investigators started with an exploratory case study approach rather than an explanatory case study approach. They began with the general hunch that the effectiveness of a work-site trip reduction program does depend on organizational culture. For efficacy, the investigators attempted to "disprove the null hypothesis"; that is, to find at least one work site that has demonstrated that the success of its trip reduction program did indeed depend on the work-site's organizational culture. The research design was based on an attempt to control for both internal and external factors to isolate the effects of management culture. This was done by selecting the individual work site as the unit of study and selecting a group of work sites located where there was comparability of external factors, including population and employment densities, alternative transportation services and amenities, parking availability, and local regulatory environment. The work sites were also selected based on likeness of internal factors, including similarity of North American Industry Classification System code and number of employees. The goal was to select a group of very similar work sites, half of which were having the most successful trip reduction performance compared against the other half having the least successful trip reduction performance. Among work sites that are similar in every respect except trip reduction performance, can we see a relationship between organizational culture and trip reduction program effectiveness? The research design also incorporated anonymity of the participants and the identities of the organizations that agreed to participate to enable all participants to talk with candor about the sensitive subject of organizational culture. 
A data collection plan was developed to incorporate the use of multiple independent sources of evidence. This creates opportunities to detect both consistencies and inconsistencies among the information explaining the effects of the trip reduction program. Sources included the extensive comparable descriptive data and trip reduction program and performance data for the work sites as collected by WSDOT, written policies and marketing materials used by the work sites, information from in-depth interviews with both ETCs and their direct supervisors, and a survey of demographic information. Additionally, a battery of feedback instruments was administered to ETCs and their supervisors by consultants with expertise in organizational effectiveness. The case study analysis of the information, including an effort to identify rival explanations (e.g., trip reduction effectiveness was explained by factors other than organizational culture), found evidence that the null hypothesis is true only sometimes. A hierarchy emerged about the level of importance of some external and internal factors. The analysis provided evidence to suggest that the organizational culture in some work sites did influence the outcome of their trip reduction programs but such influence may be overcome by other factors. For example, the organizational culture of a work site to influence trip reduction effectiveness is generally less powerful than the influence of the income level of work-site employees. Also, organizational culture and employee income level are both generally less powerful than the effect of access to high quality public transportation alternatives. Additionally, case study analysis found that the influence of the ETC is less powerful than and contingent on top and middle management support. Management support and an effective ETC are less important for a successful commuter choice program if the work site is located in an area with access to high quality public transportation alternatives and the work site employs lower income staff who must choose transportation cost savings over time savings and convenience. However, management support and an effective ETC are necessary for a successful work-site trip reduction program if the work site is not located in an area with access to high quality transportation alternatives.

With regard to the characteristics of the ETC, research results based on both the interviews and the results of the feedback instruments suggested that the ideal skill sets and personality qualities of the ETC depend on the level of maturity of the commuter choice program. For example, a new commuter choice program sponsored by a supportive yet laissez-faire top management may require a decisive ETC with leadership and problem-solving skills. An organization with a more mature commuter choice program, in which the right combination of programs, services, and incentives are already in place, may benefit most from an ETC with 
high organizational and administrative skills. Charismatic ETCs with strong interpersonal skills may be most needed and successful in commuter choice programs in which the services require outreach and "selling." A more detailed discussion of the role of the ETC is summarized in Hendricks (2005).

To review, the common anecdotes about trip reduction programs have limited use because they often lack context as well as reliable information about baseline conditions and program results. If results are reported, anecdotes may fall short of attempting to explore the possible reasons for program results.

\section{How Can This Case Study be Made Stronger? SunBank's Parking Program}

SunBank and the Downtown Orlando Transportation Management Association (DOTMA) work together to decrease the parking demand for SunBank's employees. Because only half of the roughly 1,000 employees are able to find parking in the SunBank Center garage, the DOTMA offers several TDM incentives. One program allows certain employees, including carpoolers and persons with disabilities to be placed on a waiting list for a reserved parking space in the SunBank Center garage. Once the space becomes available, SunBank pays nearly 40 percent of the employee's parking fee, including tax. Another program, the Centroplex Park ' $n$ Ride, encourages employees to park in a nearby garage that is connected to the SunBank Center via a free shuttle bus. As an added incentive to rideshare and conserve energy, SunBank offers to pay the parking fees for those employees who carpool to the Centroplex garage, but only pays 50 percent of the fees for those employees who drive alone. Source: Angela Gallogly, SunBank, Phone interview.

Reprinted from the "Commute Alternatives Systems Handbook," Center for Urban Transportation Research, University of South Florida, 1996.

The case study method described in this article differs from anecdotes in that researchers were able to reliably identify work sites with varying levels of trip reduction program performance to examine not only by comparing existing "before" (baseline performance) and "after" (program results) data, but also by identifying those work sites with programs that had a clear trend in program performance over time that were not likely due to chance or some temporary unusual 
circumstance. Unlike anecdotes, this case study method started with a research question and the formulation of a particular hypothesis, or hunch, describing an expected relationship between trip reduction program success and an influential factor. Researchers collected information from as many sources as possible and looked for relationships in the data as well as inconsistencies that may support or refute the hypothesis. Use of the case study method yielded evidence about the relationship among internal and external factors of a work site that would not have been apparent from the less complete information of an anecdote. Finally, unlike anecdotal information that is commonly used to try to persuade organization $B$ to adopt a trip reduction program similar to that of organization $A$, the expectation for use of the case study results is to discern the evidence for an apparent rule of thumb, then determine whether to apply the rule with caution to organization $B$, recognizing both the present constraints and potential opportunities afforded by organization B's unique internal and external context.

The Commuter Choice study used a more rigorous case study method for the purpose of sorting out the influence of organizational culture relative to its context. The second study, "National Smart Transportation Archive Researcher (NSTAR)," by Georggi et al. (2006), also applied the case study method but with two other purposes. The first purpose was to create an online updatable, easily searchable database of case studies documenting the impacts of TDM strategies and to begin to populate the database with case studies that supply information to enable users to make inferences with better confidence. These case studies would be for use by transportation professionals as well as those charged with developing and implementing TDM programs for work sites or other applications. Commuter choice program information and trip reduction data from more than 100 carefully selected work sites were compiled by researchers, now located on the Help Desk of the National TDM and Telework Clearinghouse, found at http://www.nctr.usf. edu/clearinghouse. These case studies are not anonymous, but include the identities of the organizations and their contact information so that TDM professionals can pursue further information. The second purpose of the NSTAR study was to provide an in-depth examination of 12 carefully selected work sites from the Help Desk, also using an exploratory case study approach. In the Commuter Choice study, we found evidence to suggest that the external condition of access to public transportation alternatives has more sway in the outcome of a trip reduction program than internal conditions of the work site. In the NSTAR study, we ask if this is always the case. Our research question is: "How important are internal work-site conditions relative to external conditions?" The hypothesis researchers 
wanted to test was "Internal work-site conditions can overcome adverse or unsupportive external conditions if the trip reduction program is explicitly supported by work-site management and the nature of the business also supports program success." The goal was to disprove the null hypothesis by finding at least one trip reduction program that demonstrates that internal conditions can overcome adverse external conditions. Disproving the null hypothesis would lend support to the proposition that work-site trip reduction programs can be successful in reducing SOV mode share, vehicle trips, and reducing VMT under the typically adverse conditions found in many urban areas of the United States.

Adverse external conditions include underdeveloped public alternative transportation options that compete poorly with SOV travel, sprawling land development patterns, abundant public parking, the lack of severe traffic congestion, and (relatively) low fuel prices. Supportive internal work-site conditions could be active in nature, such as management that demonstrably encourages trip reduction activities. Supportive internal conditions could also be passive in nature, meaning that the work site may not be consciously conducting trip reduction programs but its business activities have no intrinsic operational conflict with trip reduction activities. An example of this might be a work site that uses telework for business purposes. An operational conflict might be a business that relies on staff to travel to the offices of clients on less than one day's notice.

The research design for the NSTAR study was simplified so that other transportation professionals working on time constraints could borrow elements from this process to strengthen their case study information. The ultimate goal is to improve case study information available to the profession and encourage transportation professionals to share their information with others through submittal of data to help build case studies on the Help Desk.

The first main question that the NSTAR study addressed was to identify which work-site trip reduction programs that researchers can confidently agree demonstrate effective program results. The Help Desk archive includes 56 work sites from Washington State. The data that document their programs were selected from 1,300 work sites that participate in the Washington State Commute Trip Reduction Program. Selection of these work sites was based on a series of progressively applied criteria, including availability of data for the most recent years, availability of data spanning at least five survey periods, having a negative slope for the trend line of vehicle miles traveled (VMT), with R square greater than 0.5 , and having a negative slope for the trend line of SOV mode share, with R square greater than 0.5 . 
Having an R square greater than 0.5 means a greater level of confidence that the decrease in SOV mode share and VMT over time was not due to chance.

In addition to the development of the Help Desk case study database and the collection of case studies that confidently demonstrate effectiveness, the research design also included a more in-depth examination of 12 of the 56 outstanding work-site trip reduction programs. The 12 programs were self-selected based on ETCs willing to participate in the study. With each of the 12 work sites, researchers gathered multiple sources of evidence by conducting a brief survey, an extensive telephone interview with the ETC, and a request for any office policies, internal organization website information, brochures, and other information provided to employees about trip reduction activities, and any data that the work site collects and reports to its host municipality or other regulatory authority. Unlike the Commuter Choice study, where the aim was to compare differences in organizational culture of work sites with successful trip reduction programs to work sites with less successful trip reduction programs, the aim of the NSTAR study was to solicit the participation of work sites with a success story to tell and summarize the main internal and external factors that appeared responsible for that success. In the NSTAR study, work sites were not selected based on similar external factors but rather simply finding work sites with the best performance. As a result, examples of commute choice programs were identified that thrive in varied settings and land development patterns, including rural areas, suburban locations, and central business districts. It was found that while urban settings generally offer more commuter options, no land development pattern was shown to be absolutely necessary.

The detailed 12 case study write-ups can be found in the Best Practices Guide, located at $h t t p: / / w w w . n c t r . u s f . e d u / p d f / 77604 . p d f$. The narratives include an interpretation of the information and point out characteristics of the following.

- land use configuration of surrounding area

- public parking availability

- traffic conditions

- availability of outside support

- commuter assistance program characteristics

- conditions internal to the work site

- work site accessibility to public transit/regional accessibility 
- physical facilities

- employee characteristics

- nature of business

- organizational culture

- ETC

- graphs of change in average VMT per one-way commute

- graphs of change in percentage of employees traveling by SOVs

For example, Figures 1 and 2 illustrate the performance trends for one work site, Acordia Northwest, Inc. Figure 1 shows that the drive-alone mode share of employees decreased from 43 percent in 1993 to just 9 percent in 2003. Figure 2 indicates that AVMs traveled per employee for a one-way commute trip decreased from 7.6 miles in 1993 to 2.8 miles in 2003.

The NSTAR study sought to disprove the null hypothesis by finding at least one trip reduction program that demonstrates that internal conditions can overcome adverse external conditions, as typically found in urban areas in the United States. It is important to note that the 12 work sites were all located in an area under mandatory trip reduction requirements, which one could argue is the most supportive rather than adverse external condition for trip reduction program success. However, making trip reduction mandatory implies an environment in which some degree of difficulty and effort is required to reduce trips, otherwise a sufficient number of work sites would be reducing trips voluntarily. There is also a difference between demonstrating good faith compliance with the law and implementing a trip reduction program that achieves sustained successful results. More work sites achieve compliance but less work sites achieve program effectiveness. Case study analysis provided insight into how businesses approach the mandate. Instead of business benefits or the desire to abide by "green" business practices, the regulatory requirements did provide the primary motivation for most organizations to take action to ensure legal compliance. However, characteristics of organizational culture further determined if management believes, "If we have to do a trip reduction program, we are going to do it right and make a best effort." 


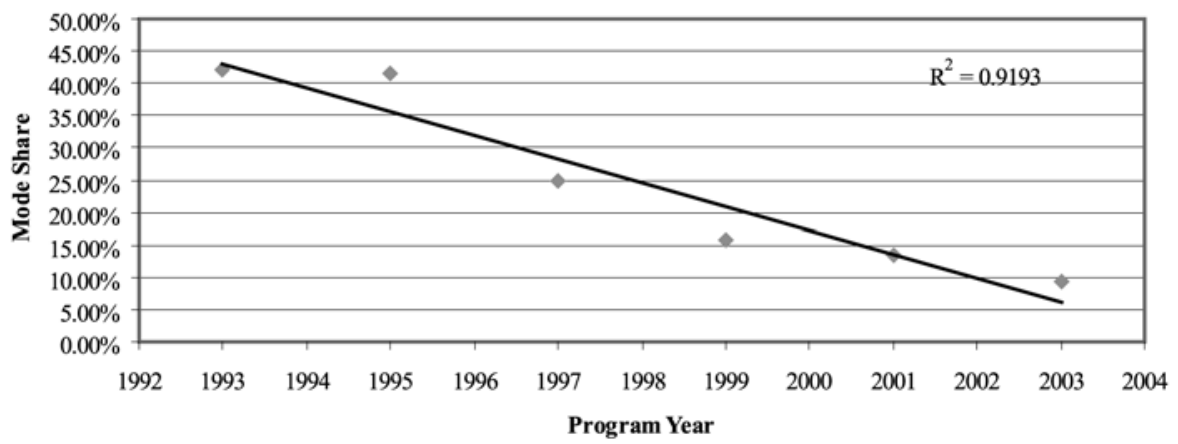

Figure 1. Percentage of Driving Alone Commuting Trip

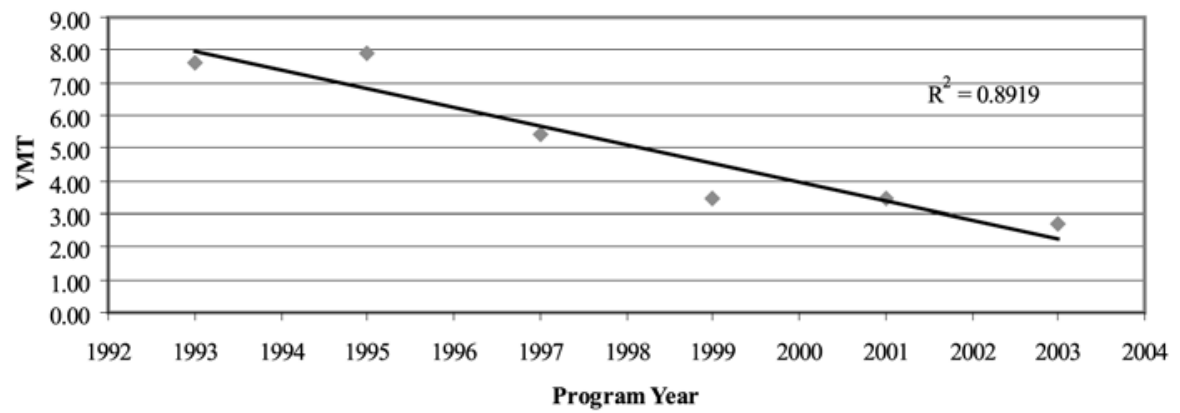

Figure 2. Vehicle Miles Traveled

\section{Presence of Supportive Management}

An analysis of the collected data and information suggested several relationships. In a mandatory regulatory environment, alignment of the nature of the business with the commute trip reduction program is not always necessary but always helpful. Where the intrinsic nature of the business might not support program success, such as a business requiring a location where public transit is not available, the presence of supportive management can still overcome external adversity to result in a successful trip reduction program. Results also found no relationship between the department in which the commuter choice program was implemented and program success. Programs can be run successfully from a number of departments, such as human resources, parking and security, payroll and benefits, 
or the office of the executive assistant, and the greater decider of success is that the staff tasked with implementing the program is sincerely interested in the program and is confident in the benefits of commuter choices. Results pointed to no specific type of administrative program oversight that is better as long as it is supportive. For example, some work sites with successful commuter choice programs are centrally managed by a remote headquarters office, while others are managed at the individual work site.

While limiting parking and leveling the playing field for parking perqs across all tiers in a work-site hierarchy sends powerful signals to employees to use transportation alternatives, study results found that some successful commuter choice programs can also tolerate some free parking. It is recognized that even superior transit service does not match the time savings and door-to-door service of driving one's own vehicle. For those organizations in which it is a business imperative to recruit the highest skilled employees, free parking is sometimes a necessary perq, and will not undermine a strong commuter choice program if the perq is applied with care. For example, one hospital that provides comprehensive commuter choice services to its employees also offers free and convenient parking to its doctors.

\section{The Intrinsic Nature of the Business}

Alternatively, in the absence of supportive management, work-site trip reduction program success might simply be due only to the intrinsic nature of the business rather than any conscious effort on the part of management and staff. Examples of this might include a lower- or moderate-income workforce that is more price sensitive to travel alternatives, or an organization that achieves business efficiencies through telework strategies.

Looking closer for other indicators that represent the intrinsic nature of businesses, no results were found to indicate that work-site commuter choice program performance was linked to work-site industry type or size. In addition, no results were found to indicate that any employee type is necessary to commuter choice program success. The case studies demonstrated that successful commute trip reduction programs serve diverse workforces, such as blue-collar unionized employees and white-collar professionals. 


\section{Recommendations for Action}

\section{For TDM Marketing Professionals}

The Commuter Choice study provided some insights for TDM professionals who want to focus marketing efforts on more receptive organizations. They should target organizations that have some combination of the following attributes:

- work-site location has access to good quality transit

- large support staff for whom cost of transportation is more important than time savings and convenience

- most of the affected employees remain in an office setting during the workday

- most of the affected employees usually work routine predictable hours

- business motivations of work sites to support trip reduction activities beyond regulatory compliance, including organizations that:

- generate environmental hazards, and also may search for ways to demonstrate to the public that they are good stewards of the environment

- want an image that they are "green," progressive, or that they are motivated by "doing what is right"

- have short-term transportation benefits that would enhance employee recruitment and retention

- feel a responsibility to take a leadership role and set the standard for positive action (primarily government)

- are exploring long-term profitability within the context of environmental, social, and financial sustainability practices

TDM marketing professionals may also want to evaluate the usefulness of case study information for demonstrating the causes of trip reduction program outcomes. Based on the NSTAR study, the checklist given in Figure 3 provides an outline for evaluation. Few case studies contain all this information, but the more reliable information, the better. 
Identification of information source

Credible source

Multiple independent sources of information that show consistency

Contact information for the source

Recently updated

Measurements of travel behavior prior to TDM strategy implementation (baseline)

Measurements of travel behavior after TDM strategy implementation

Continued periodic measurements of travel behavior over time (performance trend)

Monitoring of baseline and travel behavior change of same individuals

Detailed information about work-site characteristics

TDM program characteristics

Changes

Employee characteristics

Changes

External environment

Changes

__ Regulatory

Changes

Physical

Changes

Services

Changes

Economic

Changes

Other

Changes

Selection of performance indicators that adequately reflect goals and purposes of stakeholders

Performance indicators that are properly measured

Interpretation of the data

Addresses relationship between TDM strategy and any resulting change in travel behavior Describes other possible causes of changes in travel behavior (rival explanations)

Provides a balanced presentation (what works, what has not worked)

\section{Figure 3. Checklist of TDM Case Study Quality}

\section{For Work Sites}

Several actions for improving work-site commuter choice programs were identified from the results of both case study research projects, as summarized below.

1. Locate the work site with high quality transportation alternatives. Locate near other important offices, institutions, and retail that are complementary 
to the business instead of acres of parking lots and garages that isolate your business from customers.

2. Provide political support to the local transit agency by telling local elected officials that businesses want public transit service for their employees.

3. Offer a transit subsidy and other incentives to employees.

4. Select an ETC that is managerial level with direct communications access to top management decision-makers and has influence on decisions relating to the trip reduction program budget.

5. Incorporate activities of the trip reduction program into the job description of the selected ETC and allocate a realistic amount of time to their execution.

6. Make explicit in the guiding principles the role that the trip reduction program plays in the organization, so that trip reduction and promoting commute alternatives become part of the organizational culture.

7. Consider removing any parking subsidies or related perks to those higher in the organization or at least offer the same parking subsidy to everyone regardless of position in the organization.

\section{For TDM Researchers}

The case studies for both research projects described above used employee data aggregated to the employer level. As a result, reductions in SOV mode share or reductions in VMT are net reductions across all employees of a work site. An advantage of the aggregated data is that it protects the privacy of individual employees. A disadvantage of the aggregated data is that researchers are unable to track the change in travel behavior of individuals over time. In the future, with the informed consent of employees who agree to participate in travel behavior research, combined with emerging technologies for collecting detailed travel behavior data of individuals, we can gain better insight into the reasons why people travel as they do as well as stronger evidence of the impact of trip reduction programs relative to other contextual factors that influence travel behavior.

The NSTAR study found that in a mandatory regulatory environment, alignment of the intrinsic nature of the business with the commute trip reduction program is not necessary but helpful. For future research, a hypothesis could be that in a voluntary environment, the converse is true: An alignment of the intrinsic nature of the business is necessary for the business to involve itself with commute trip reduction program activities. The evolution of business practices to incorporate 
environmental, financial, and social sustainability objectives for long-term profitability may further recognize employee travel as part of these considerations.

\section{Conclusions}

The NSTAR study provided evidence to disprove the null hypothesis by evaluating 56 case studies in which VMT and SOV decreased over time and for which the R square value indicated a high level of confidence that these decreases were not random. These work sites were subject to varying degrees of adverse external conditions associated with underdeveloped transit systems, sprawling land development, abundant parking, the lack of severe traffic congestion, and (relatively) low fuel prices. However, the hypothesis that "Internal work site conditions can overcome adverse or unsupportive external conditions if the trip reduction program is explicitly supported by work site management and the nature of the business also supports program success" is likely overspecified. Examples were found in which trip reduction program effectiveness can be achieved with either management support or a naturally aligned business. Furthermore, no factors were found to be always absolutely necessary, unless in the absence of others.

Each work site is a case study in shades of gray, in which the importance of a factor depends not only on its magnitude or intensity but also its presence in combination with other factors. With regard to management support of trip reduction programs, the results demonstrate the adage, "Where there's a will, there's a way" but also that there is great leeway in how a work site can assemble a program to achieve effectiveness. TDM professionals can stimulate creativity and encourage effective results by describing the variety of strategies for achieving performance, then giving the work site the latitude to craft its unique approach to achieve a performance target.

\section{Endnote}

${ }^{1}$ After 2002, the name for the U.S. General Accounting Office was changed to the U.S. Government Accountability Office. 


\section{References}

Chen, Qiuzi, W. Patrick Beaton, and H. Meghdir. 1995. Profile of employee transportation coordinators. Transportation Research Record 1496. Washington, DC: Transportation Research Board, National Research Council, National Academy Press, 123-130.

Georggi, Nevine L., Sara J. Hendricks, Liren Zhou, and Christopher Hagelin. 2006. National smart transportation archive researcher. Funded by the Florida Department of Transportation and the Federal Transit Administration through the National Center for Transit Research. Prepared by the Center for Urban Transportation Research, University of South Florida, Tampa, FL.

Hendricks, Sara J. 2005. Effectiveness of programs for work site trip reduction: The influence of organizational culture. Transportation Research Record 1924. Washington, DC: Transportation Research Board of the National Academies, pp. 207-214.

Hendricks, Sara J., and Ajay Joshi. 2004. Commuter choice program case study development and analysis. Funded by the Florida Department of Transportation and the Federal Transit Administration through the National Center for Transit Research. Prepared by the Center for Urban Transportation Research, University of South Florida, Tampa, FL.

Lopez-Aqueres, Waldo. 1993. Conceptual framework to study the effectiveness of employer trip-reduction programs. Transportation Research Record 1404. Washington, DC: Transportation Research Board, National Research Council, National Academy Press, pp. 55-63.

Martin, Joanne. 2002. Organizational culture: Mapping the terrain. Thousand Oaks, CA: Sage Publications.

United States General Accounting Office. 1990. Case study evaluations. GAO Program Evaluation and Methodology Division, GAO/PEMD-91-10.1.9. November.

Wachs, Martin, and Genevieve Giuliano. 1992. Employee transportation coordinators: A new profession in Southern California. Transportation Quarterly (July): 411-428.

Winters, Philip L., and Rafael A. Perez. 2004. Worksite trip reduction model and manual. Funded by the Florida Department of Transportation and the Fed- 
eral Transit Administration through the National Center for Transit Research. Prepared by the Center for Urban Transportation Research and the Computer Science and Engineering Department, University of South Florida, Tampa, FL. Yin, Robert K. 1981. The case study crisis: Some answers. Administrative Science Quarterly 26 (March): 58-65.

\section{About the Authors}

SARA J. Hendricks (hendricks@cutr.usf.edu) is a senior research associate at the Center for Urban Transportation Research, University of South Florida. She graduated with a Master of Regional Planning degree from the University of North Carolina at Chapel Hill. She formerly worked as a planner for Orth-Rodgers \& Associates in Philadelphia and Howard Needles Tammen Bergendoff in Raleigh, North Carolina.

NeVine LABIB GeORGG (georggi@cutr.usf.edu) is a senior research associate at the Center for Urban Transportation Research, University of South Florida. She graduated with a Master of Science in Civil Engineering degree from the University of South Florida. She formerly held positions as a transportation planner with DSA Group, Inc. in Tampa and as a transportation research engineer at the Energy Research Center at Cairo University, Egypt. 